\title{
3 Research Soure \\ Identification of Circulating miR-210 as a Promising Biomarker for Lung Adenocarcinoma
}

\section{Jinru Xue}

China-Japan Union Hospital of Jilin University

Hua Xin ( $\nabla$ xhua@jlu.edu.cn )

https://orcid.org/0000-0001-6144-1908

Erna Jia

China-Japan Union Hospital of Jilin University

Na Ren

China-Japan Union Hospital of Jilin University

\section{Research article}

Keywords: lung adenocarcinoma, circulating, miRNAs, biomarker

Posted Date: July 3rd, 2019

DOI: https://doi.org/10.21203/rs.2.10912/v1

License: (c) (i) This work is licensed under a Creative Commons Attribution 4.0 International License. Read Full License 


\section{Abstract}

Background: Lung adenocarcinoma (LUAD), with an increasing morbidity and mortality globally, is the most common histological subtype of lung cancer (LC). Early diagnosis of LUAD can significantly prolong survival time. Multiple researches have revealed that circulating miRNAs might be used as promising biomarkers for early detection of LUAD Material and Methods: A meta-analysis was conducted to find circulating miRNAs for early diagnosis of LUAD and summarized their diagnostic values based on GEO database. Further, by bioinformatics analysis we predicted the target genes of circulating miRNAs owning statistically significant, and explored the molecular regulation mechanisms and biological processes of the target genes in the pathogenetic of LUAD. Results: overall, six datasets were eligible, information on original expression levels of circulating miR-21, miR-155, miR-210, miR-126, miR-486, miR182 , and miR-17 extracted independently by two reviewers. Finally, we found circulating miR-210 had good diagnostic efficacy for LUAD detection, the combined AUC value was 0.83 . We then predicted 480 overlapped genes of miR-210 at least 5 of 11 databases. Bioinformatics analysis showed statistically significant GO analysis 38 items and KEGG pathways 21 items. Response to hypoxia may be the mainly involved biological process of miR-210 in LUAD. Nine hub genes (FBXO, FBXL, MGRN1, ATG7, CUL3, RAB, ADAMTS, SEMA, THBS2) were obtained by construct PPI network. Conclusions: our study implied that circulating miR-210 may be a promising noninvasive biomarker for early detection of LUAD, but further researches are needed to validate the promising results and to identify specific biological processes and target genes.

\section{Background}

Lung cancer (LC) is a major cause of cancer-related death worldwide, and the annual incidence and mortality are about 2.1 million and 1.8 million, respectively [1, 2]. The most common histological subtype is lung adenocarcinoma (LUAD), accounting for $82.6 \%$ of non-small cell lung cancer (NSCLC) [3] and $40 \%$ $\sim 50 \%$ of LC [4]. Moreover, during the last several years the prevalence of LUAD has been increasing globally, especially in women, young patients and non-smokers $[3,5]$, and the morbidity of young patients even reaches $57.5 \%-77.9 \%$ [3, 6-11]. The 5-years survival rate of LUAD is approximately $15 \%$ [12], yet which in stage $\triangle$ LUAD patients can achieve 100\% [13-15]. At present, the methods widely used for LC diagnosis, such as sputum exfoliation cytology, x-ray, and CT scan do not improve the early diagnostic rate or distinguish LUAD from other tumor subtypes. Therefore, an early accurate diagnosis for LUAD is an extremely urgent need and a crucial approach to enhance patient's chances to receive a proper treatment for improving the survival rate in clinical [16].

Circulating miRNAs as noninvasive effective diagnostic markers for NSCLC have recently became a major research area [17-20], and whether can be used as highly specific diagnostic biomarkers for LUAD have attracted more attentions. miRNAs are the broadest class of gene regulatory molecules in biological processes. The expression profile of miRNAs reflect the tumor development lineage and differentiation stages, is related to the clinicopathological features of tumor subtypes [21,22], and can identify tumor histological subtypes based on origin, histology, invasiveness, and chemical sensitivity [23-25]. For the 
past few years, several researches have verified that circulating miRNAs can be used for early diagnosis of LUAD with good diagnostic efficiency [26-30], and many researchers have been working hard on the role of miRNAs in the pathogenesis of LUAD [31-33]. But inconsistences of miRNAs profiles existed among studies, due to the differences of the specimen, ethnicity, pre-analysis preparations, and other influencing factors [34-37]. So far, miR-21, miR-155, miR-210, miR-126, miR-486, miR-182, and miR-17 are the circulating miRNAs with most reported frequency and great diagnostic efficiency for NSCLC [18, 3841]. Whether those circulating miRNAs can accurately identify LUAD and are predicted to apply in clinical practice is still inconclusive, and the specific biological processes and molecular regulatory mechanisms of miRNAs in LUAD are also undefined.

In present study, we performed a systematic summary of the published datasets based on the GEO database which investigated circulating miRNAs for LUAD detection. The primary purpose was to analysis the diagnostic performance of circulating miR-21, miR-155, miR-210, miR-126, miR-486, miR-182, and miR-17 for LUAD. Second, we explored the biological processes and molecular regulatory mechanisms of the aforementioned miRNAs with statistically significant in LUAD development by bioinformatics analysis.

\section{Methods}

Selection of GEO dataset and data extraction A systematic literature search was applied to identify studies assessing circulating miRNAs as diagnostic biomarkers for LUAD. We mined the GEO database for eligible articles until Apr 26, 2019. The search strategy as follows: ((lung OR pulmonary) AND (cancer OR carcinoma OR tumour OR tumor OR malignanc* OR neoplas* OR nodule OR adenoma*)) AND (microRNA* OR miRNA* OR miR*)). And we used the following filters to filter the search results again: "Homo sapiens"[porgn] AND "gse"[Filter]. The initial screening was to browse the title, using the following exclusion criteria: (1) non-related to NSCLC, (2) non-human studies, (3) non-related to the topic. The second screening used the following exclusion criteria: (1) the studies about the miRNAs in tissue, bronchial epithelium, and lung fibroblasts. (2) without health control group or relevant data. Two reviewers independently screened the involved microarray datasets, extracted the expression levels of miR-21, miR-155, miR-210, miR-126, miR-486, miR-182, and miR-17 used for diagnosis LUAD.

Subsequently, controversies from the two reviewers were resolved by consensus.

Statistical analysis The RevMan software version 5.3 and MetDiSc software version 1.4 were used to perform statistical analyses in this study. In addition, sensitivity, specificity, and AUC values of circulation miRNAs for LUAD were calculated by MedCalc software version 18. Standardized mean difference (SMD) was used to assess the relationship between circulating miRNAs expression levels and LUAD. A fixedeffect model was adopted to merge SMD when the heterogeneity was low $\left(I^{2} \varangle 50 \%\right.$, or $\left.\mathrm{P} \otimes 0.1\right)$. Otherwise, a random-effect model was used [42]. Eatimaed $S D$ by the fomula: $S D_{\text {estimated }} \approx f \times$ range [43]. $P \leq 0.05$ was 
considered statistically significant. Bar plot was performed using statistical software R x64 (version 3.5.3).

Target prediction analysis and bioinformatic analysis we extracted the predicted target genes in 11 miRNA databases (miRWalk, Microt4, miRanda, mirbridge, miRDB, miRNAMap, Pictar2, PITA, RNA22, RNAhybrid, Targetscan), and the genes overlapped in at least 5 of 11 databases were selected as target genes. Gene ontology (GO) and Kyoto Encyclopedia of Genes and Genomes (KEGG) enrichment analysis of overlapped genes were analyzed by online DAVID database (Pख0.05 and FDR $₫ 1.5$ was considered statistically significant). Furthermore, we used the online STRING database to construct protein-protein interaction (PPI) network analysis (combined score冈0.9 was considered statistically significant) and used MCODE plugin of Cytoscape to identify the hub genes.

\section{Results}

Results from the GEO database Finally, six microarray datasets (concluded 115 LUAD patients and 96 healthy controls) were eligible for this study (Figure 1), and the original expression levels of circulating miR-21, miR-155, miR-210, miR-126, miR-486, miR-182 and miR-17 were supplied in supplement material 1. Compared with healthy controls, meta-analysis showed SMD of miR-210 in LUAD patients had statistically significant, $S M D$ value was $1.86(95 \% \mathrm{Cl}(3.52,0.20), \mathrm{P}=0.03)$. The forest plot was presented in Figure 2. SMD of miR-21, miR-155, miR-126, miR-486, miR-182, and miR-17 had no statistically significant, the forest plots were shown in supplement material 2 . The heterogeneity result of circulating miR-210 was severe $\left(I^{2}=94 \%, P \otimes 0.00001\right.$, Figure 2$)$. The pooled sensitivity, specificity and AUC values of miR-210 were $0.83,0.60$ and 0.78 , respectively. SROC curve was depicted in Figure 3.

GO and KEGG analyses A total of 480 overlapped genes were obtained, all predicted target genes included in 11 miRNA databases were displayed in supplement material 3. Predicting functional annotation information of overlapping genes by GO enrichment analysis, as shown in Figure 4, GO-BP included 24 statistical items, the top three enriched items were response to hypoxia, positive regulation of chemokine biosynthetic process, and leukocyte migration; GO-MF included 10 statistical items, the top three enriched items were protein complex binding, protein kinase activity, and transcription factor activity, sequence-specific DNA binding; GO-CC included 4 statistical items, the top three enriched items were extracellular matrix, integral component of plasma membrane, and focal adhesion. The statistically significant KEGG enrichment pathways were 21 items, the main enrichment pathways were pathways in cancer, Chagas disease (American trypanosomiasis), and TNF signaling pathway, as shown in Figure 4.

PPI network analysis PPI network contained 480 nodes and 239 edges, $p=0.0138$. The protein network diagram was presented in Figure 5. Used MCODE plugin of Cytoscape identified 3 significant modules 
and nine hub genes: FBXO, FBXL, MGRN1, ATG7, CUL3, RAB, ADAMTS, SEMA, THBS2, shown in Figure 6. The standard settings as follows: Degree Cutoff $=2$, Node Score Cutoff $=0.2, \mathrm{~K}-\mathrm{Core}=2$, Max. Depth=100.

\section{Discussion}

Based on the six eligible microarray datasets, we compared the expression levels of circulating miR-21】

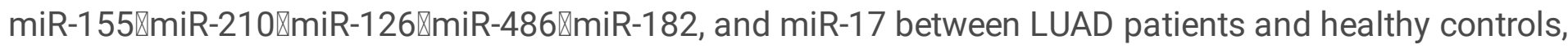
and found that miR-210 was the only statistically significant circulating miRNA with excellent diagnostic performance for LUAD (AUC value was 0.83). Then we elucidated the oncogenic role of miR-210 in LUAD through bioinformatics analysis. We identified 38 statistically significant GO items and 21 KEGG pathways. Consistent with previous researches, we again proved that hypoxia was an important feature in LUAD and miR-210 was regarded as the most important hypoxemir associated with the tumorigenesis of LUAD. We also constructed PPI network of the target genes and identified nine hub genes involved. Therefore, this study provided evidences for circulating miR-210 as a promising noninvasive biomarker for early LUAD detection, and provided a foundation for further researches on circulating miR-210 in the pathogenetic of LUAD.

MiR-210 is one of the most widely studied miRNAs and has captured great attention since it has been shown to be associated with various biological processes and the development of different human diseases thus far [44]. The increasing literatures exploring the role of circulating miR-210 in LUAD have been proved miR-210 to be a noninvasive biomarker for LUAD detection [45-47]. In Tamiya H's research, it revealed the AUC value of exosomes miR-210 in the diagnosis of LUAD combined with pleural effusion was 0.81 [48]. In Y. HE's study, circulating miR-210 combined with other miRNAs (miR-199a-3p, miR-148a3p, miR-378d, miR-138-5p) had a diagnostic specificity of $90.2 \%$ in LUAD presenting with pulmonary nodules [49]. Also, Shen et al used plasma miRNAs panel (miR-210, miR-21, miR-486-5p, and miR-126) for the detection of LUAD which yield $92 \%$ sensitivity and $97 \%$ specificity [50]. Not long ago, one research demonstrated that serum miR-210 displayed considerable accuracy in discriminating LUAD patients from healthy controls, the AUC value was 0.84 [46]. With the consistent of previous results, this study showed the combined AUC value of circulating miR-210 for LUAD were 0.83 . Theoretically, circulating miR-210 test was a promising method to diagnosis LUAD in this study. It provides a novel approach for improving management of LUAD, which is a major subtype of NSCLC, and finding efficacy noninvasive detection markers to classify NSCLC subtypes. Thus, future validation on circulating miR-210 as a noninvasive specific biomarker for LUAD should preferably be done in the context of large scale prospective studies.

Overall $\varangle$ we demonstrated that the diagnostic performance of circulating miR-210 for LUAD appeared to be rather promising. However, there was severe heterogeneity among studies reported circulating miR-210 as biomarkers for LUAD in this meta-analysis (Figure 2). One of the heterogeneity is that the variation of sample types and population characteristics among studies. The miRNA profiles are differences, which were abtained form different sample types and different sample preparation [34, 51]. Although plasma, serum and whole blood are all origin of blood-based samples, reports described the variations circulating miRNA profile by sample handling and preparation $[52,53]$, and in which the major factor of concern is 
the blood fractionation protocol $[51,54,55]$. Population characteristics, such as age, smoking status, and ethnicity, have been demonstrated as the other potential source of miRNA level variability in LC patients $[37,56,57]$. Another important source of heterogeneity was the small size sample in eligible datasets. Three of six datasets have small size sample (GSE93300, GSE94536, GSE111803). Sample size is one of the critical elements affecting the statistical power, and large population researches can help to reduce analytical bias and to improve diagnostic performance. In this study, one dataset (GSE103149) only uploaded the averaged normalized values without the original expression levels of circulating miRNAs and in which SD values were estimated by estimation formula. It may be one more factor of the heterogeneity. Considering the limitations mentioned above, developing new constantly technologies and bioinformatics tools to reduce the analysis bias are necessary.

The roles of miRNAs in the initiation and progression of LUAD is with complicated gene expression and signaling pathways, researchers worldwide have been focus on the theme recent years [27, 31, 58, 59]. In this study, we predicted the target genes of miR-210 in LUAD as well, and then used bioinformatics analysis to explore the potential biological processes and molecular pathogenesis of the target genes involved. And the results showed that nine central genes were identified (FBXO, FBXL, MGRN1, ATG7, CUL3, RAB, ADAMTS, SEMA, THBS2). In GO annotation and KEGG pathway analysis, the results revealed that responsing to hypoxia was the mainly involved biological process, the major site of biological process was extracellular matrix, protein complex binding was the dominating molecular function, and pathways in cancer was the significant pathway aggregating most genes. In solid tumors, oxygen concentration is reduced variously in which hypoxia is the most common neoplastic microenvironment. A current overview shows miR-210 has been identified as a major miRNA induced under hypoxia, and plays numerous crucial roles in the cellular response to hypoxia, such as in apoptosis [60], angiogenesis [61], cell cycle regulation [62], and mitochondrial metabolism [63]. Furthermore, several studies have suggested a direct connection between miR-210 and hypoxia, specially carrying the HIF-1a-binding site in its promoter [64-66]. While miR-210 has garnered interest as a prospective biomarker for LUAD detection, further work is require to confirm its detailed role in biological processes and molecular mechanisms.

\section{Conclusions}

Our study suggested that circulating miR-210 had great potential to be used as a noninvasive diagnostic biomarker for LUAD. Compared to previous reviews, we focused on studies identified to LUAD patients from health controls based on the original expression levels of circulating miR-210 in order to improve early diagnosis rate. We also analyzed the possible source of heterogeneity among eligible datasets in this study. In particular, we rediscovered that response to hypoxia may be the mainly involved biological process of miR-210 in LUAD and predicted nine hub genes through bioinformatics analysis. However, the specific role of hub genes is still not fully known. Furthermore, a big set of samples prospective and molecular biology researches are needed to validate the diagnostic efficacy of circulating miR-210 for LUAD detection and to search for specific biological processes and target genes of LUAD. 


\section{Abbreviations}

FBXO: member F-box protein family, FBXL: member F-box and leucine rich repeat protein family, MGRN1: mahogunin ring finger 1, ATG7: autophagy related 7, CUL3: cullin 3, RAB: member RAS oncogene family, ADAMTS: member ADAM metallopeptidase with thrombospondin type 1 motif family, SEMA: semaphorin, THBS2: thrombospondin 2. SD: standard deviation, SEN: Sensitivity, SPE: Specificity, SROC: Summary receiver operating characteristic, AUC: The area under the SROC curve. MiRNA: MicroRNA.

\section{Declarations}

Ethics approval and consent to participate囚Not applicable.

Consent for publication $₫$ Not applicable.

Availability of data and material $\mathbb{T}$ The dataset supporting the conclusions of this article is included within the article and its additional file.

Competing interests $₫$ The authors declare no competing interest

Funding $₫$ None.

Authors' contributions: HX and JRX designed this study. JRX, HX and ENJ collected literatures and conducted the analysis of pooled data. NR helped to draft the manuscript.

JRX wrote the manuscript. All authors contributed to review the manuscript. All authors read and approved the final manuscript.

Acknowledgements $₫$ Not applicable

\section{References}

1. Allemani, C., et al., Global surveillance of trends in cancer survival 2000-14 (CONCORD-3): analysis of individual records for 37513025 patients diagnosed with one of 18 cancers from 322 populationbased registries in 71 countries. Lancet, 2018. 391(10125): p. 1023-1075.

2. Bray, F., et al., Global cancer statistics 2018: GLOBOCAN estimates of incidence and mortality worldwide for 36 cancers in 185 countries. CA Cancer J Clin, 2018. 68(6): p. 394-424.

3. Hsu, C.L., et al., Advanced non-small cell lung cancer in patients aged 45 years or younger: outcomes and prognostic factors. BMC Cancer, 2012. 12: p. 241.

4. Zappa, C. and S.A. Mousa, Non-small cell lung cancer: current treatment and future advances. Transl Lung Cancer Res, 2016. 5(3): p. 288-300.

5. Torres-Duran, M., et al., Residential radon and lung cancer in never smokers. A systematic review. Cancer Lett, 2014. 345(1): p. 21-6. 
6. Ak, G., et al., Lung cancer in individuals less than 50 years of age. Lung, 2007. 185(5): p. 279-286.

7. Chen, K.Y., et al., Distribution according to histologic type and outcome by gender and age group in Taiwanese patients with lung carcinoma. Cancer, 2005. 103(12): p. 2566-74.

8. Kuo, C.W., et al., Non-small cell lung cancer in very young and very old patients. Chest, 2000. 117(2): p. 354-7.

9. Liam, C.K., K.H. Lim, and C.M. Wong, Lung cancer in patients younger than 40 years in a multiracial Asian country. Respirology, 2000. 5(4): p. 355-61.

10. Subramanian, J., et al., Distinctive characteristics of non-small cell lung cancer (NSCLC) in the young: a surveillance, epidemiology, and end results (SEER) analysis. J Thorac Oncol, 2010. 5(1): p. 23-8.

11. Zhang, J., et al., Multicenter analysis of lung cancer patients younger than 45 years in Shanghai. Cancer, 2010. 116(15): p. 3656-62.

12. Meng, F., et al., The genomic alterations of lung adenocarcinoma and lung squamous cell carcinoma can explain the differences of their overall survival rates. J Cell Physiol, 2018.

13. Kadota K, S.K., D'Angelo SP, et al. , Validation of the proposed IASLC/American Thoracic Society (ATS)/European Respiratory Society (ERS) international multidisciplinary classification of lung adenocarcinoma (ADC). 2011. 6: p. 244-85.

14. Yoshizawa, A., et al., Impact of proposed IASLC/ATS/ERS classification of lung adenocarcinoma: prognostic subgroups and implications for further revision of staging based on analysis of 514 stage I cases. Mod Pathol, 2011. 24(5): p. 653-64.

15. YoshizawaA, S.S., MoreiraAL, et al., Validation of the IASLC/ATS/ERS lung adenocarcinoma (ADC) classification and use of comprehensive histologic subtyping (CHS) for architectural gradingin432Japanese patients. . Mod Pathol 2011. 24: p. 429A.

16. Blandin Knight, S., et al., Progress and prospects of early detection in lung cancer. Open Biol, 2017. $7(9)$.

17. Han, Y. and H. Li, miRNAs as biomarkers and for the early detection of non-small cell lung cancer (NSCLC). J Thorac Dis, 2018. 10(5): p. 3119-3131.

18. He, Y., et al., Current State of Circulating MicroRNAs as Cancer Biomarkers. Clin Chem, 2015. 61(9): p. 1138-55.

19. Heegaard, N.H., et al., Circulating micro-RNA expression profiles in early stage nonsmall cell lung cancer. Int J Cancer, 2012. 130(6): p. 1378-86.

20. Mozzoni, P., et al., Plasma and EBC microRNAs as early biomarkers of non-small-cell lung cancer. Biomarkers, 2013. 18(8): p. 679-86.

21. 26229396Lu, J., et al., MicroRNA expression profiles classify human cancers. Nature, 2005. 435(7043): p. 834-8.

22. Peng, S., et al., Multi-class cancer classification through gene expression profiles: microRNA versus mRNA. J Genet Genomics, 2009. 36(7): p. 409-16. 
23. Hollis, M., et al., MicroRNAs potential utility in colon cancer: Early detection, prognosis, and chemosensitivity. World J Gastroenterol, 2015. 21(27): p. 8284-92.

24. Kishikawa, T., et al., Circulating RNAs as new biomarkers for detecting pancreatic cancer. World J Gastroenterol, 2015. 21(28): p. 8527-40.

25. Zaporozhchenko, I.A., et al., The potential of circulating cell-free RNA as a cancer biomarker: challenges and opportunities. Expert Rev Mol Diagn, 2018. 18(2): p. 133-145.

26. Feng, M., et al., Upregulated Expression of Serum Exosomal microRNAs as Diagnostic Biomarkers of Lung Adenocarcinoma. Ann Clin Lab Sci, 2018. 48(6): p. 712-718.

27. Sun, Y., et al., Circulating microRNA-339-5p and -21 in plasma as an early detection predictors of lung adenocarcinoma. Pathol Res Pract, 2018. 214(1): p. 119-125.

28. Zhou, X., et al., A six-microRNA panel in plasma was identified as a potential biomarker for lung adenocarcinoma diagnosis. Oncotarget, 2017. 8(4): p. 6513-6525.

29. Rani, S., et al., Global analysis of serum microRNAs as potential biomarkers for lung adenocarcinoma. Cancer Biol Ther, 2013. 14(12): p. 1104-12.

30. Wang, Y., et al., Identification of a three-miRNA signature as a blood-borne diagnostic marker for early diagnosis of lung adenocarcinoma. Oncotarget, 2016. 7(18): p. 26070-86.

31. Geng, Y., et al., Identification of crucial microRNAs and genes in hypoxia-induced human lung adenocarcinoma cells. Onco Targets Ther, 2016. 9: p. 4605-16.

32. Kim, M.H., et al., Discriminating between Terminal-and Non-Terminal Respiratory Unit-Type Lung Adenocarcinoma Based on MicroRNA Profiles. PLoS One, 2016. 11(8): p. e0160996.

33. Lin, K., et al., MicroRNA expression profiles predict progression and clinical outcome in lung adenocarcinoma. Onco Targets Ther, 2016. 9: p. 5679-5692.

34. McDonald, J.S., et al., Analysis of circulating microRNA: preanalytical and analytical challenges. Clin Chem, 2011. 57(6): p. 833-40.

35. Foye, C., et al., Comparison of miRNA quantitation by Nanostring in serum and plasma samples. PLoS One, 2017. 12(12): p. e0189165.

36. Badrnya, S., R. Baumgartner, and A. Assinger, Smoking alters circulating plasma microvesicle pattern and microRNA signatures. Thromb Haemost, 2014. 112(1): p. 128-36.

37. Huang, R.S., et al., Population differences in microRNA expression and biological implications. RNA Biol, 2011. 8(4): p. 692-701.

38. Jiang, M., et al., Clinically Correlated MicroRNAs in the Diagnosis of Non-Small Cell Lung Cancer: A Systematic Review and Meta-Analysis. Biomed Res Int, 2018. 2018: p. 5930951.

39. Yang, Y., et al., The clinical use of circulating microRNAs as non-invasive diagnostic biomarkers for lung cancers. Oncotarget, 2017. 8(52): p. 90197-90214.

40. Yu, H., et al., Circulating microRNA biomarkers for lung cancer detection in Western populations. Cancer Med, 2018. 7(10): p. 4849-4862. 
41. Yu, N., et al., A meta-analysis: microRNAs' prognostic function in patients with nonsmall cell lung cancer. Cancer Med, 2017. 6(9): p. 2098-2105.

42. Ioannidis, J.P., N.A. Patsopoulos, and E. Evangelou, Uncertainty in heterogeneity estimates in metaanalyses. BMJ, 2007. 335(7626): p. 914-6.

43. Kelley, G.A. and K.S. Kelley, Statistical models for meta-analysis: A brief tutorial. World J Methodol, 2012. 2(4): p. 27-32.

44. Bavelloni, A., et al., MiRNA-210: A Current Overview. Anticancer Res, 2017. 37(12): p. 6511-6521.

45. Xie, S., et al., miR-210 promotes lung adenocarcinoma proliferation, migration, and invasion by targeting lysyl oxidase-like 4. J Cell Physiol, 2019. 234(8): p. 14050-14057.

46. Switlik, W.Z., et al., Serum miR-210-3p as a Potential Noninvasive Biomarker of Lung Adenocarcinoma: A Preliminary Study. Genet Test Mol Biomarkers, 2019. 23(5): p. 353-358.

47. Cui, H., et al., Tissue inhibitor of metalloproteinases-1 induces a pro-tumourigenic increase of miR210 in lung adenocarcinoma cells and their exosomes. Oncogene, 2015. 34(28): p. 3640-50.

48. Tamiya, $\mathrm{H}$., et al., Exosomal MicroRNA Expression Profiling in Patients with Lung Adenocarcinomaassociated Malignant Pleural Effusion. Anticancer Res, 2018. 38(12): p. 6707-6714.

49. He, Y., et al., Serum microRNAs improving the diagnostic accuracy in lung cancer presenting with pulmonary nodules. J Thorac Dis, 2018. 10(8): p. 5080-5085.

50. Shen, J., et al., Plasma microRNAs as potential biomarkers for non-small-cell lung cancer. Lab Invest, 2011. 91(4): p. 579-87.

51. Duttagupta, R., et al., Impact of cellular miRNAs on circulating miRNA biomarker signatures. PLoS One, 2011. 6(6): p. e20769.

52. Mitchell, P.S., et al., Circulating microRNAs as stable blood-based markers for cancer detection. Proc Natl Acad Sci U S A, 2008. 105(30): p. 10513-8.

53. Turchinovich, A., et al., Characterization of extracellular circulating microRNA. Nucleic Acids Res, 2011. 39(16): p. 7223-33.

54. Blondal, T., et al., Assessing sample and miRNA profile quality in serum and plasma or other biofluids. Methods, 2013. 59(1): p. S1-6.

55. Pritchard, C.C., et al., Blood cell origin of circulating microRNAs: a cautionary note for cancer biomarker studies. Cancer Prev Res (Phila), 2012. 5(3): p. 492-497.

56. Takahashi, K., et al., Cigarette smoking substantially alters plasma microRNA profiles in healthy subjects. Toxicol Appl Pharmacol, 2013. 272(1): p. 154-60.

57. Noren Hooten, N., et al., Age-related changes in microRNA levels in serum. Aging (Albany NY), 2013. 5(10): p. 725-40.

58. Daugaard, I., et al., The association between miR-34 dysregulation and distant metastases formation in lung adenocarcinoma. Exp Mol Pathol, 2017. 102(3): p. 484-491.

59. Li, Y.J., et al., miR-218 suppresses epithelial-to-mesenchymal transition by targeting Robo1 and Ecop in lung adenocarcinoma cells. Future Oncol, 2017. 13(28): p. 2571-2582. 
60. Kulshreshtha, R., et al., A microRNA signature of hypoxia. Mol Cell Biol, 2007. 27(5): p. 1859-67.

61. Huang, X. and J. Zuo, Emerging roles of miR-210 and other non-coding RNAs in the hypoxic response. Acta Biochim Biophys Sin (Shanghai), 2014. 46(3): p. 220-32.

62. Giannakakis, A., et al., miR-210 links hypoxia with cell cycle regulation and is deleted in human epithelial ovarian cancer. Cancer Biol Ther, 2008. 7(2): p. 255-64.

63. Chen, Z., et al., Hypoxia-regulated microRNA-210 modulates mitochondrial function and decreases ISCU and COX10 expression. Oncogene, 2010. 29(30): p. 4362-8.

64. Huang, X., Q.T. Le, and A.J. Giaccia, MiR-210-micromanager of the hypoxia pathway. Trends Mol Med, 2010. 16(5): p. 230-7.

65. Mathew, L.K. and M.C. Simon, mir-210: a sensor for hypoxic stress during tumorigenesis. Mol Cell, 2009. 35(6): p. 737-8.

66. Ivan, M. and X. Huang, miR-210: fine-tuning the hypoxic response. Adv Exp Med Biol, 2014. 772: p. 205-27.

\section{Figures}




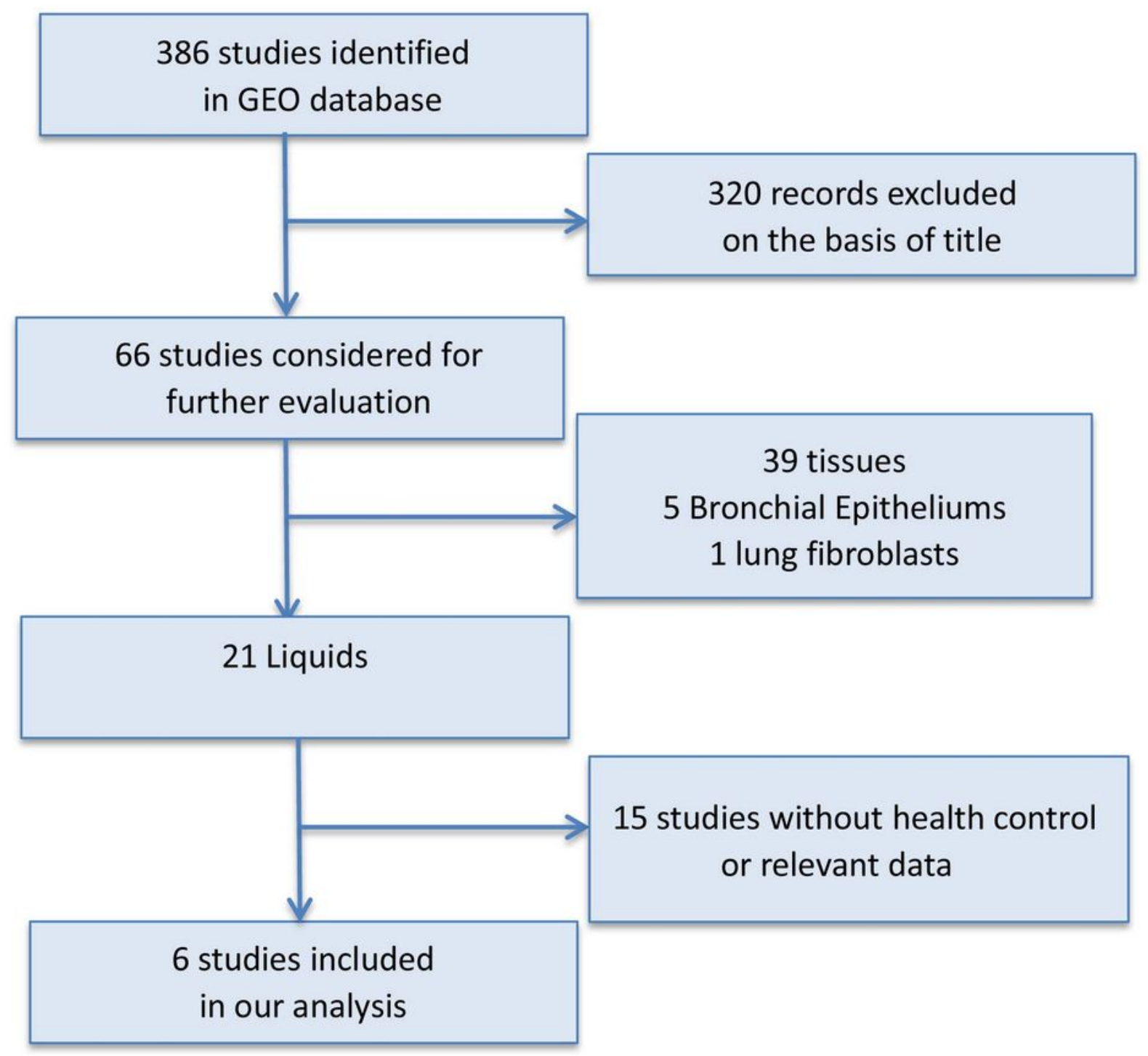

Figure 1

Overview of the literature search process (up to 26th of Apr 2019). 


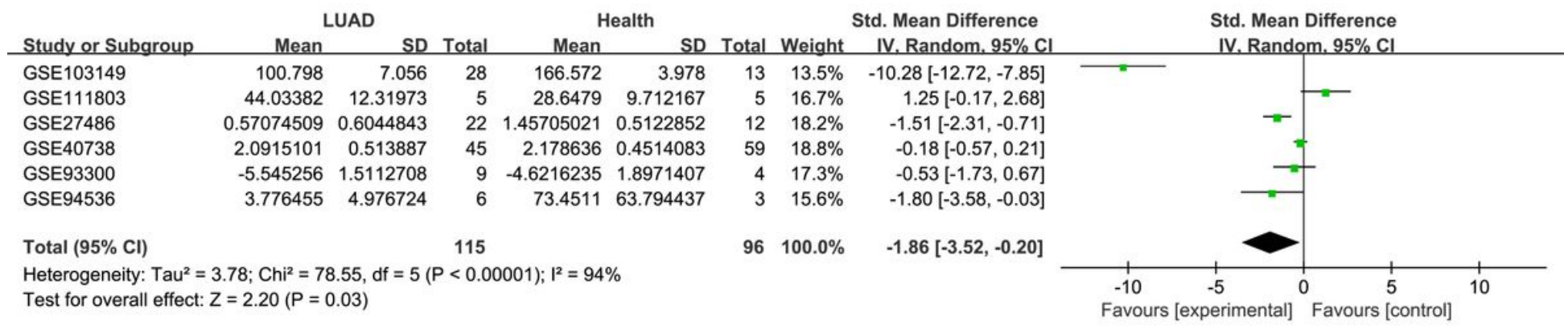

\section{Figure 2}

Forest plot determining the differences in circulating miR-210 by meta-analysis (a represents estimated $\mathrm{SD})$. 


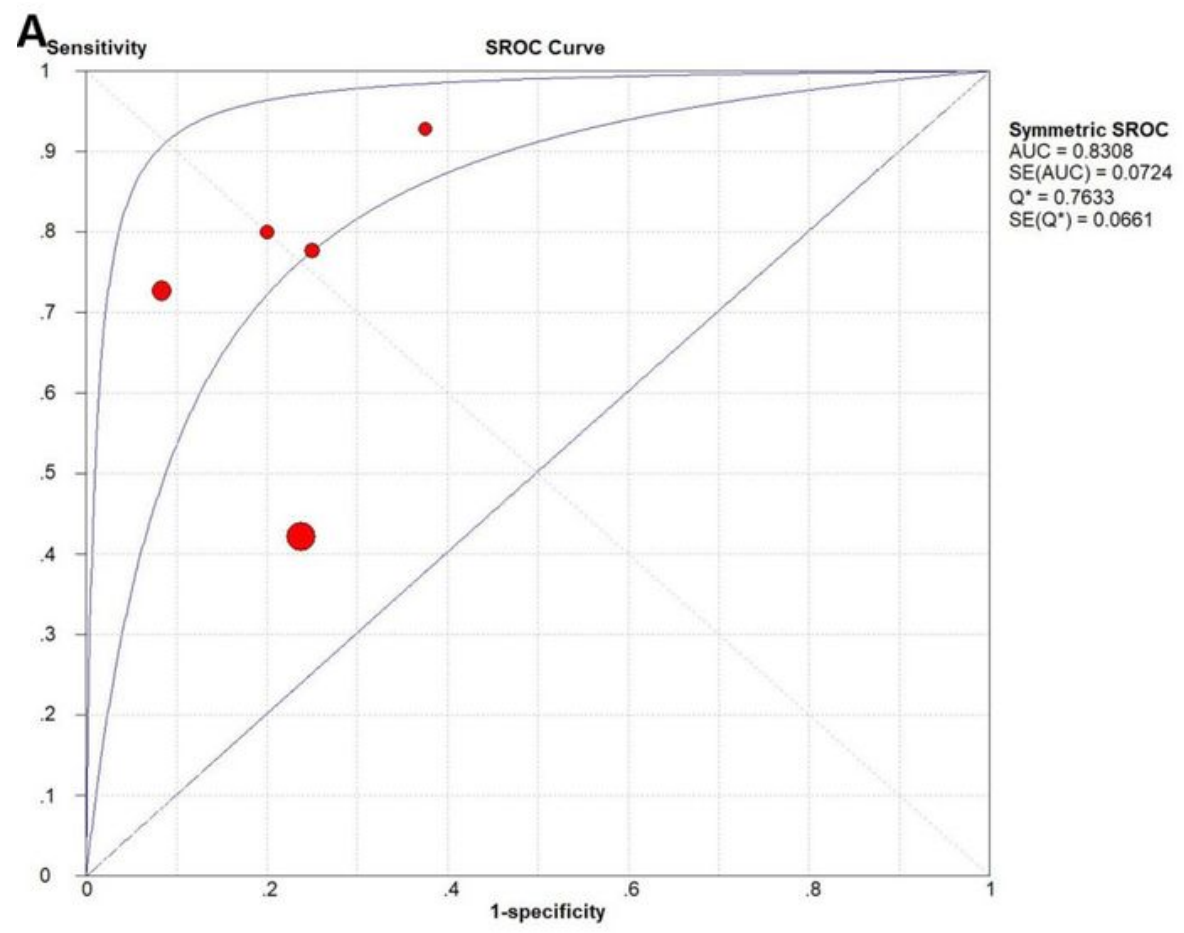

B

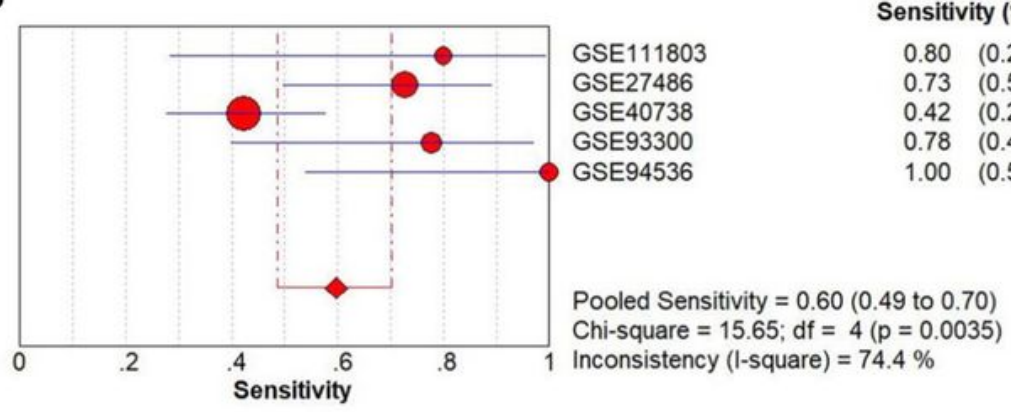

C

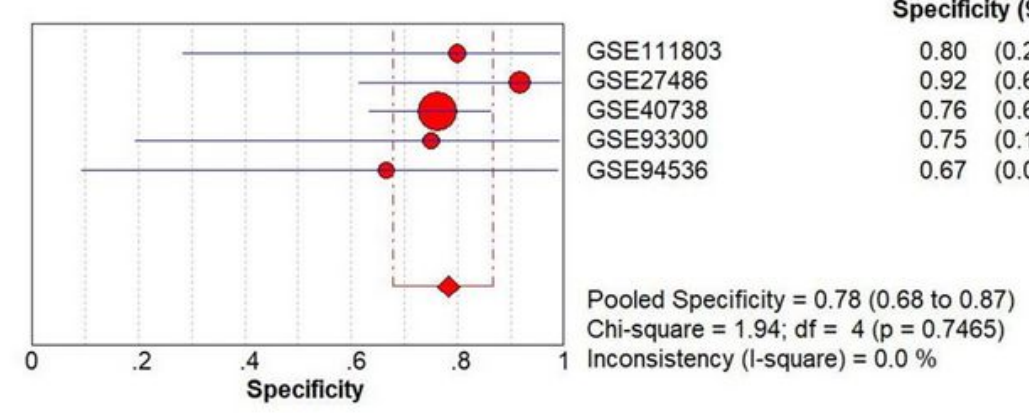

Specificity $(95 \% \mathrm{Cl})$

$0.80 \quad(0.28-0.99)$

$0.92(0.62-1.00)$

$0.76 \quad(0.63-0.86)$

$\begin{array}{lll}\text { GSE93300 } & 0.75 & (0.19-0.99) \\ \text { GSE94536 } & 0.67 & (0.09-0.99)\end{array}$

Pooled Specificity $=0.78(0.68$ to 0.87$)$

Chi-square $=1.94 ; \mathrm{df}=4(\mathrm{p}=0.7465)$

Specificity

\section{Figure 3}

Diagnostic performance of circulating miR-210 for LUAD detection. (A) SROC curve of circulating miR210, red dot represents included dataset; (B) Pooled sensitivity of circulating miR-210; (C) Pooled specificity of circulating miR-210. 


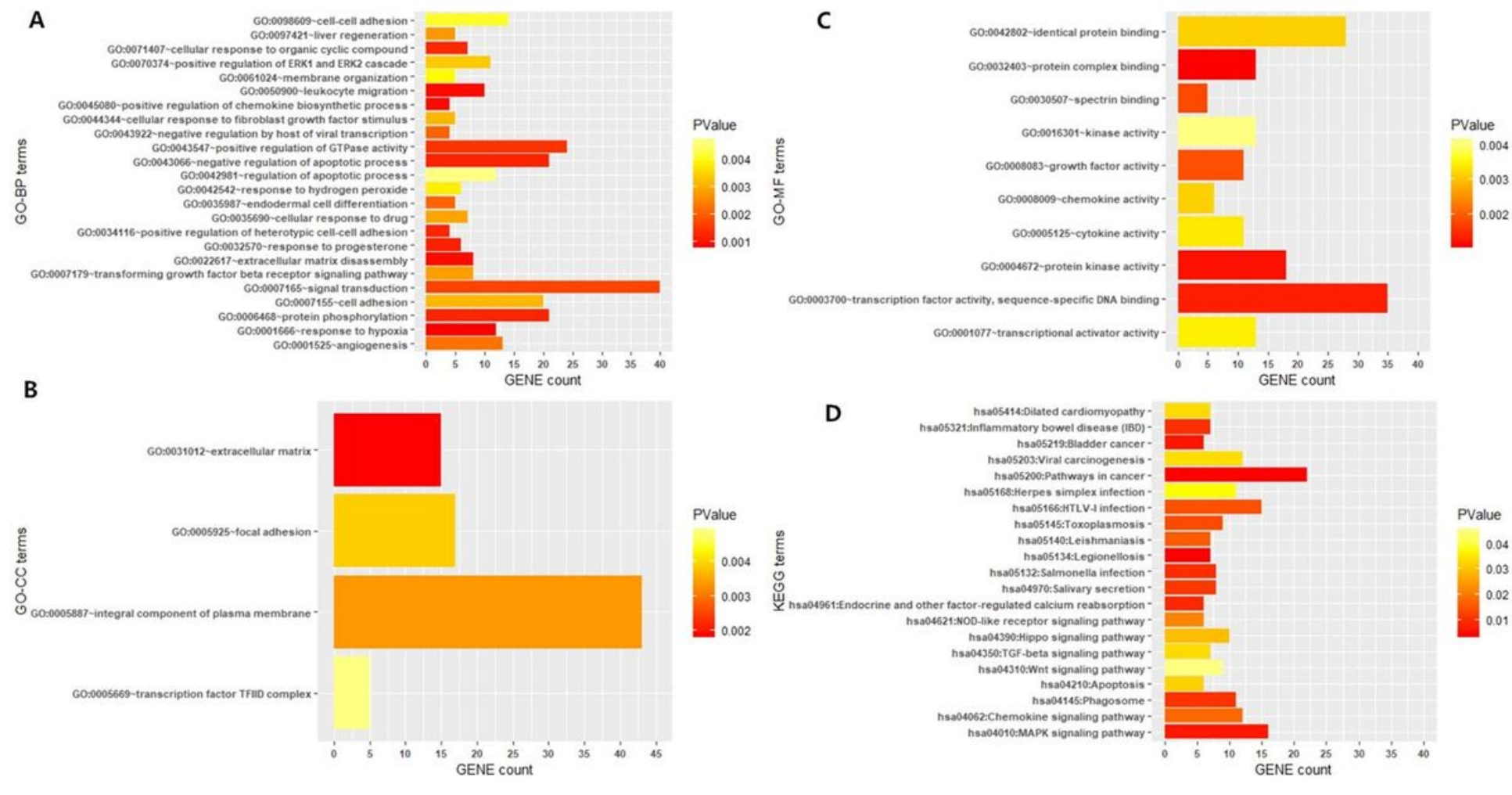

\section{Figure 4}

Significantly enriched GO and KEGG terms for the 480 overlapping genes. (A) The significantly enriched BP terms. (B) The significantly enriched CC terms. (C) The significantly enriched molecular function (MF) terms. (D) The significantly enriched KEGG terms. 


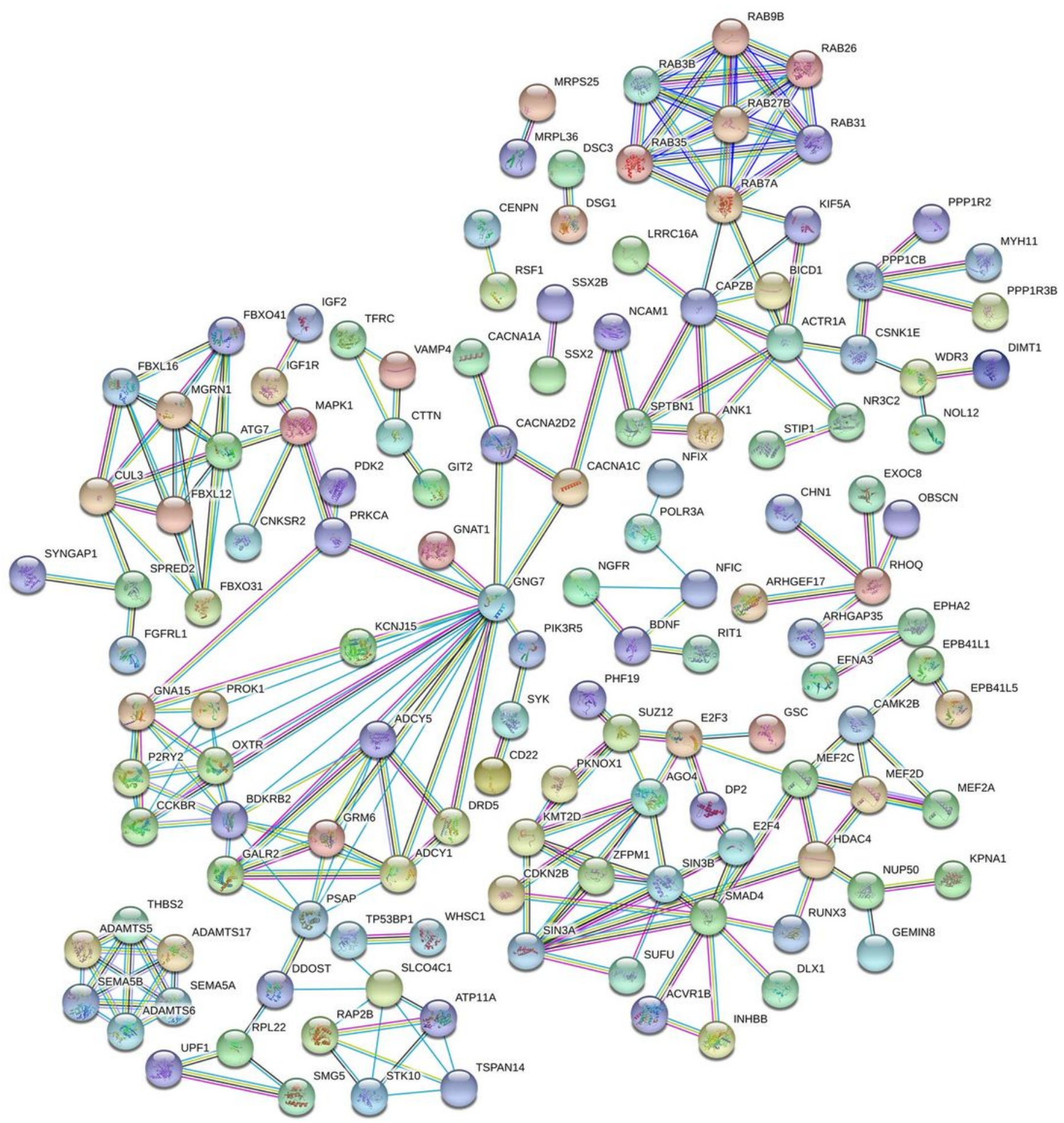

Figure 5

PPI network of the overlapped LUAD-related genes. 

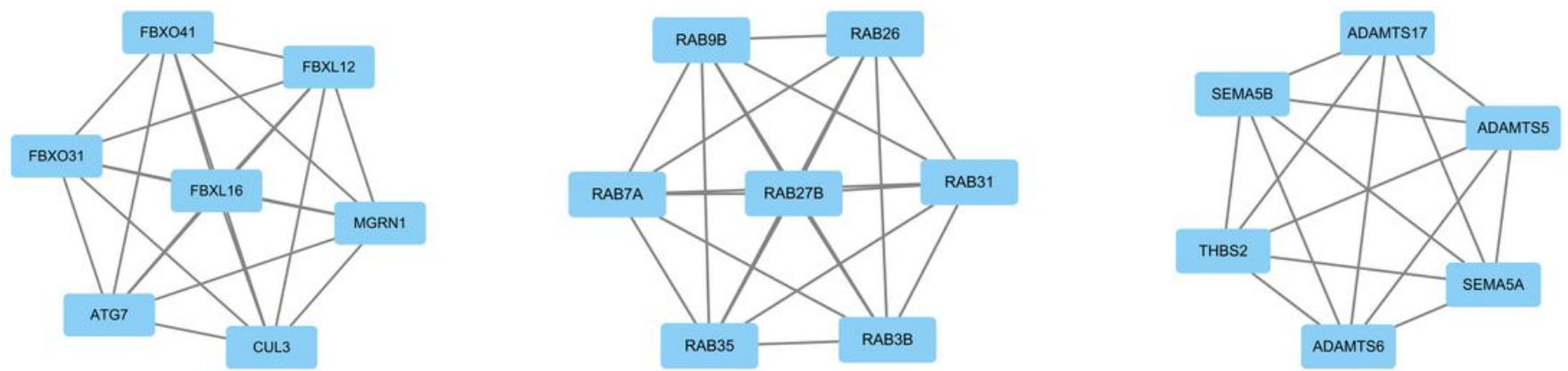

Figure 6

The top 3 modules and hub genes from the PPI network.

\section{Supplementary Files}

This is a list of supplementary files associated with this preprint. Click to download.

- Forestplot.jpg

- theoriginalexpressionofcirculaitingmiRNAs.xlsx

- allpredictedtargetgenes.xlsx 